\title{
A Study of the Clinical Profile of Cerebral Venous Sinus Thrombosis
}

\author{
Dr Vishwanath K*, Dr K P Balaraj**, Dr. B D Chavan*** \\ *Assistant professor, **professor department of medicine kims Bangalore. ***professor jjmmc davangere
}

\begin{abstract}
Background and objectives: CVT is an important cause of a stroke. Its varied presentations make the clinician to have strong degree of suspicion and investigate accordingly thus instituting early treatment.

Objectives: To study clinical profile of cortical venous sinus thrombosis and to assess if possible any prognostic factors.

Methods: Total 30 patients diagnosed as CVT by CT scan/MR angio (Direct and indirect findings of CVT) above 18 years studied. Patients with less than 18 years and stroke of arterial origin were excluded.

Results: The male to female ratio was 1:5, and total of 23 patients were alive with age group of 21-35 being maximally involved. Puerperium formed the bulk (18 patients) in female patients and of which 10 were from headache (94\%), convulsions (78\%) were predominant symptoms. Lateralising signs like hemiplegia (34\%), were seem. Papilledema was an important sign present in 50\% of patients. Superior sagital sinus thrombosis (40\%) was the most common sinus to be involved with early diagnosis and treatment $68 \%$ patients were discharged without deficits rest had some deficits.

Interpretation and Conclusion:

1) Puerperium formed the bulk of cause of CVT in female patients.

2) Headache convulsions, neurological defects and papilledema formed the important quadrant of presentation of CVT.

3) Superior sagittal sinus was the commonest involved.

4) With adequate treatment many had good recovery.

Key words: Cerebral venous thrombosis; puerperium; anticoagulation.
\end{abstract}

\section{Introduction}

Cerebral venous sinus thrombosis is an important cause of stroke specially in peripartum setting and stroke in young.

The earliest reference to CVT appeared in 1825 in the French literature with postmortem confirmation in a 45 years old man with headache. Seizure, confusions wide spread malignancy.

Earlier considered to be a rare entity; over the past 30 years the outlook for CVT has been dramatically improved, by the advent of sensitive neuro imaging technique, and increasing awareness of variety of presentations and effective treatment for the same has helped its cause

\section{OBJECTIVES}

To study the clinical profile of cerebral venous sinus thrombosis and to assess if possible any prognostic factors

1) Source of Data:

\section{Methodology}

Patients admitted in Chigateri General Hospital and Bapuji Hospital attached to J.J.M Medical College, Davangere.

A total of thirty patients admitted in hospital during the study period who had clinical and CT/MR angio, features (direct and indirect) suggestive of cerebral sinus venous thrombosis were selected

Inclusion criteria:

- All patients aged $>18$ years.

- An acute or sub acute neurological illness in a patient under appropriate clinical condition whose CT features (direct and indirect signs) /MR angio where ever done suggestive of cerebral venous sinus thrombosis.

- The examination should have suggested constellation of signs of raised ICT /Neurological deficits / seizures.

Exclusion criteria:

- Patients $<18$ years

- Stroke due to arterial thrombosis, haemorrhage and patients with ICSOL. 
All the patients selected were subjected to detailed history, clinical examination and investigated.

1) Complete blood picture

2) Blood sugar, blood urea serum creatinine

3) CSF Analysis (wherever deemed necessary)

4) Serology for HIV $-1 \& 2$

5) Urine culture and sensitivity.

\section{HISTORY AND EXAMINATION:}

All patients selected were asked a detailed history with emphasis to find out common etiologies for CVT. In patients of puerperium further detailed assessment of the parity, antenatal illness, post natal sepsis, fever, dehydration were enquired.

A general physical examination and a detailed neurological assessment with other systems were done to look for any evidence of etiologies.

III. Results

TABLE 1: TOTAL MORTALITY

\begin{tabular}{|l|l|l|}
\hline Total patients of CVT & Alive & Dead \\
\hline 30 & 23 & 07 \\
\hline
\end{tabular}

In this study of 30 patients 23 were alive and 7 patients died.

TABLE 2: AGE DISTRIBUTION

\begin{tabular}{|l|l|l|}
\hline Age(year) & Number of patients & percentage \\
\hline $18-20 \mathrm{Y}$ & 5 & $17 \%$ \\
\hline $21-35 \mathrm{Y}$ & 15 & $49 \%$ \\
\hline$>35 \mathrm{Y}$ & 10 & $34 \%$ \\
\hline
\end{tabular}

The maximum number of patients was in the age group of 21-35, the youngest was 18 years old

The clustering of patients in these age groups probably was the result of puerperium as one of the commonest prothrombotic state associated with CVT

TABLE 3: SEX RATIO

\begin{tabular}{|l|l|l|}
\hline Total & Male & Female \\
\hline 30 & 5 & 25 \\
\hline
\end{tabular}

In our study the ratio was 1:5

TABLE 4: NUMBER OF PATIENTS WITH PUERPERIUM AS A MAJOR CAUSE AND THEIR PARITY

\begin{tabular}{|l|l|l|l|}
\hline Total Number & Puerperium & Alive & Dead \\
\hline 30 & TOTAL 18(Primi 10) & 16 & 2 \\
\hline
\end{tabular}

Thus puerperium formed the bulk of causes of CVT and here $60 \%$ of the cases were primi. Two patients had not attended antenatal checkup.

TABLE 5: INITIAL SYMPTOMS

\begin{tabular}{|l|l|l|}
\hline Symptoms & No & Percentage \\
\hline Headache & 28 & $94 \%$ \\
\hline Convulsions & 21 & $70 \%$ \\
\hline Altered sensorium & 20 & $68 \%$ \\
\hline & & \\
\hline
\end{tabular}




\begin{tabular}{|l|l|l|}
\hline Focal deficits & 13 & $43 \%$ \\
\hline Fever & 10 & $33 \%$ \\
\hline
\end{tabular}

Thus headache was the universal presenting symptom followed by convulsions, focal deficits altered sensorium and fever.

Neurological Examination:

IV. Examination Findings:

TABLE 6: LEVEL OF CONSCIOUNESS AT TIME OF PRESENTATION

\begin{tabular}{|l|l|l|l|}
\hline Level of consciousness & Total & Alive & Dead \\
\hline Fully conscious & 8 & 8 & - \\
\hline Drowsy & 15 & 13 & 3 \\
\hline Unconscious with purposive movements & 4 & 2 & 2 \\
\hline Deeply comatose & 3 & - & 3 \\
\hline
\end{tabular}

Thus mortality was high in patients who presented with deep coma.

TABLE 7: FOCAL DEFICITS/NEUROLOGICAL SIGNS

\begin{tabular}{|l|l|l|}
\hline Signs & Total & \% \\
\hline Hemiplegia & 11 & 34 \\
\hline Aphasia & 07 & 22 \\
\hline Papilledema & 18 & 60 \\
\hline
\end{tabular}

Thus lateralising signs like hemiparesis was seen in $34 \%$ of case and papilledema was the most prominent sign. Aphasia was also a common finding and one patient had Wrenickes aphasia. Majority of patients had nominal aphasia especially during recovery.

INVESTIGATIONS:

TABLE 8: HAEMOGLOBIN PERCENTAGE

\begin{tabular}{|l|l|l|l|}
\hline Hb\% & Number of patients & Patients alive & Dead \\
\hline$<5$ & - & - & - \\
\hline $5-8$ & 09 & 07 & 02 \\
\hline $8.1-10$ & 04 & 02 & 02 \\
\hline$>10$ & 17 & 14 & 03 \\
\hline
\end{tabular}

Of the total, of 25 patients were anemic though the total number of deaths appears to be more with $\mathrm{Hb}>10 \mathrm{gm} \%$, the percentage of mortality was higher when there was moderate to severe anemia.

TABLE 9: C.S.F ANALYSIS

\begin{tabular}{|l|l|}
\hline C.S.F changes & No \\
\hline Normal & 03 \\
\hline Protein rise & 02 \\
\hline Polymorphic pleocytosis & 04 \\
\hline Lymphocytic pleocytosis & 01 \\
\hline Hemorrhagic CSF with xanthochromia & 01 \\
\hline
\end{tabular}


11 patients were subjected to CSF analysis whenever there was a suspicion of Meningitis of which 5 were normal and abnormality seen in rest eight with polymorphic $\mathrm{pl}$ pleocytosis being the maximum.

TABLE 10: CT SCAN FINDINGS

\begin{tabular}{|l|l|l|}
\hline Sinus involved & No & Percentage \\
\hline Sagittal sinus thrombosis & 12 & $40 \%$ \\
\hline Hemorrjagic venous infarction & 05 & $16 \%$ \\
\hline Venous infarction & 08 & $26 \%$ \\
\hline Straight sinus thrombosis & 03 & $10 \%$ \\
\hline Cavernous sinus thrombosis & 2 & $08 \%$ \\
\hline
\end{tabular}

TABLE 11: NEUROLOGIAL STATE AT TIME OF DISCHARGE

\begin{tabular}{|l|l|}
\hline No deficits & $68 \%$ \\
\hline Minimal hemiparesis & $20 \%$ \\
\hline Continuing deficits & $12 \%$ \\
\hline
\end{tabular}

The total leucocyte count was done in all patients. The TLC was increased whenever there was a suspicion of meningitis and LP analysis was done blood chemistry and urine routine was done in all patients. Blood sugar was normal in all patients except two where it was in high normal range.

Blood urea and creatinine was also done in all patients three patients had significantly rised blood urea serum creatinine values.

Two of them had developed prerenal failure after acute gastroenteritis which had lead to severe dehydration and prone for thrombosis a phenomenon more common in children than adults.

One patient where meningitis was suspected local infection were ruled out and as she was subjected to antiphospholipid antibody test but was normal.

Seven patients died in this group

\section{PROGNOSIS:}

The recovery pattern in the rest is as follows:

a) Sensorium: of the total 15 alive patients who presented with altered sensorium 7

managed to recover within seven days completely

b) Convulsion : all patients presenting with convulsion responded well to anticonvulsants.

c) Neurological signs at the time of discharge is as shown.

d) Of the patents who had minimal deficits only $50 \%$ could be followed up for 6 months

( due to constraint of time and non compliance by few patients). Among them only $30 \%$

Had improved, rest had same deficits. Those who had continuing deficits at time of discharge, only $30 \%$ patients came up for follow-up of which only $25 \%$ had improved rest continued to have same deficits.

\section{Discussion}

In this study totally 30 cases were studied of which 23 were alive and 7 patients died.Study done by (Mehta SR et al ${ }^{1}$ ) of the 43 patients 2 died. In the present study 7 patients died.

\section{Sex ratio :}

Observations by various series have revealed (Agarwal DS et $\mathrm{al}^{2} \mathrm{M}: \mathrm{F}$ 1:1.38)

(Mehta SR et al m:I 1:1.4). ${ }^{1}$ Thus various rations have been obtained.

In this study it was 1:5. The superior methods of diagnosis (MR Angiography and better investigation facilities in suspected case of CVT employed by them has enabled their studies a better and correct sex ratio than the present study. Though our study is similar with increased female ratio, the discrepancy is more due to the lacuna of the present study.

\section{Age distribution :}

Comparing the age group involved $20-35$ was the commonest age group involved In various series ( Mehta SR et al $77.8 \%$ ) (Ameri et al ${ }^{3}-11$ - patients $61 \%$ ) 
The present study also showed similar findings with $49 \%$ in the same age group and $35 \%$

Were in age $>35$ years.

This can be attributed to peripartum being very common cause in our setup. In patients >35 years, infections and dehydration following acute GE and were the other reasons

found to be more common.

\section{Parity:}

Kallbag and Woolf (1967) ${ }^{4}$ wrote that there probably exists increasing risk of developing CVT with increasing parity. Dubosis (1956) found a ratio of 23:18 for primi to others. The results of present study showed $60 \%$ of cases with puerperium were primi (primi 60\%) is concurrent with Dubosis series.

\section{Symptoms and clinical findings:}

The symptomatology and clinical findings depends on speed of thrombosis and site of thrombosis. Headache appeared to be common in cerebral venous thrombosis, varying from $29 \%$ to $77.8 \%$ in the present study headache was seen in $94 \%$ of patients.

TABL: 12: COMPARISON OF PATIENTS WITH HEAD ACHE

\begin{tabular}{|l|l|l|}
\hline Authors & Number of cases & Percentage with headache \\
\hline Mehta. SR, et al (2002) & 45 & $77.8 \%$ \\
\hline Nagaraja et $\mathrm{al}^{5}(1989)$ & 78 & $71.8 \%$ \\
\hline Kalbag and Woolf(1967) & 34 & $29 \%$ \\
\hline Present study & 30 & $94 \%$ \\
\hline
\end{tabular}

The manifestations that indicate the cerebral cortical involvement like convulsions and paralysis closely follow headache.

Carrol et $\mathrm{al}^{6}$ (1966) found convulsions in $29.83 \%$ of patients Srinivasan and $\operatorname{Natarajan}^{7}(1974)$ found convulsions in $66 \%$ abd Mehta .SR et al found convulsions in $26.6 \%$. In this present $70 \%$ of patients had convulsion of which generalized tonic clonic type was commonest.Paralysis was a common findings and usually followed convulsive phenomenon especially

In puerperal group ( $\operatorname{King}^{8}$ 1960) Hemiplegia was the commonest form noticed in various series (Prakash $\mathrm{c}$ and sing $^{9}$ 1960, Singh and padmavathi 1961) ${ }^{10}$. The present study had $34 \%$ patients with hemiplegia.Of the quadrat of presentations of CVT, papilledema is an important sign and was seen in various series. Found in $20 \%$ Srinivasan and Natarajan (1974) in 16.6\%. Mehta et al (2002) in 77.8\%). In this present study it was found in $50 \%$ cases.

\section{Investigations}

Anemia has often been noted and has been a Hallmark sign of puerperal CVT (Singh and Padmavathi 1961) ${ }^{10}$, similar findings has been noted in the present study.

The investigative procedures like leucocyte count, blood sugar did not contribute much to the diagnosis and were non specific.

Except two cases where the blood urea levels were high where patients ha developed pre renal failure after acute GE. This had a definite contribution to prognosis of these patients.

In patients where risk factor like puerperium and meningitis were excluded only one patient could be investigate for antiphospholipid antibody syndrome and others it was not possible for economic reasons. Due to lack of facilities like platelet adhesive index and fibrinogen level estimation, these tests could not be done.

\section{CSF Analysis:}

The most important contribution of CSF analysis is to rule out meningitis to if present to know the type of meningitis

TABLE 13 :CSF ANALYSIS

\begin{tabular}{|l|l|l|l|}
\hline $\begin{array}{l}\text { Author }- \\
\text { no of cases }\end{array}$ & Xanthochromia & Protein rise & Pleocytosis \\
\hline $\begin{array}{l}\text { Krayenbuhl } \\
11(1967)-55\end{array}$ & 10 & 14 & 13 \\
\hline $\begin{array}{l}\text { Jolly et } \\
\text { al(1971)- } \\
36\end{array}$ & 08 & 13 & - \\
\hline
\end{tabular}




\begin{tabular}{|l|l|l|l|}
\hline $\begin{array}{l}\text { Present } \\
\text { study }-13\end{array}$ & 01 & 02 & 05 \\
\hline
\end{tabular}

- MR angiography is the gold standard investigation for case of suspected CVT but due to problem of availability and financial constraints this study, enrolled many CT proven cases.

Of which superior sagittal sinus thrombosis $(40 \%)$ was the commonest other findings included hemorrhagic venous infarction (16\%) cavernous sinus thrombosis $(8 \%)$ straight sinus thrombosis (10\%). Agarwal DS et al in their study had noted that sagittal sinus was affected in $52 \%$ while sigmoid in $22 \%$.

\section{Conclusion}

CVT is an important cause of stroke especially in the peripartum settings and stroke in young, yet it is grossly under recognized.

Thrombosis of cerebral venous sinus may occur in absence of demonstrable cause, may occur in settings of hematologic disorder/ coagulation abnormality or may result from local contiguous infections process. Apart from it diverse causes, its varied presentations necessitate one to have a strong degree of suspicion in appropriate context. Once the diagnosis is made with early treatment like anti edema measures, judicious use of herparin it has a good prognosis.

\section{SUMMARY}

This study comprised of 30 patients diagnosed to have cerebral sino venous thrombosis. The observations that were made are:

- The illness was seen maximum in age group of 21 to 35 typified by the fact that puerperium forms an important causes of CVT.

- The females puerperiun predominated and in males meningitis was very common .

- In patients with puerperium as the cause, primi formed the bulk of them.

- Headache was a universal phenomenon followed by convulsions, altered sensorium and fever.

- Papilledema was important sign, Hemiplegia and aphasia was also noted in few patients.

- LP whenever done showed in majority a polymorphic pleocytosis, with rise in proteins.

- Sagittal sinus thrombosis was the commonest sinus involved. In two patients cavernous sinus thrombosis was seen.

- Mortality was less, the out come and follow up gave better results as there was judicious use of heparin, anti convulsants and anti edema measures.

\section{References}

[1]. Mehta et al.editors. abstracts of $59^{\text {th }}$ annual conference of API:2004Jan18-21:Hyderabad:JAPI\{ 51 $\} 2003 . P 1196$

[2]. Agarwal et al. editors. Abstracts of $59^{\text {th }}$ annual conference of API:2004Jan1821:Hyderabad:JAPI $\{51\} 2003 . P 12-79$

[3]. Ameri CA,BousseMG.cerebral venous thrombosis.Neuralclinic1992;10:87-111

[4]. KalbaghRM,Woolf AL,editors.cerebral venous thrombosis.London: university press; 1967

[5]. Nagaraja D,Taly AB,puerperal cerebralsino venous thrombosis.progress in clinical neuro science1986;165-177

[6]. Carrol JD,Lead D and Lee HA.Cerebral thrombo phlebitis in pregnancyand puerperium.QJ med :35:347

[7]. SrinivasanK,Natarajan M.Cerebral Venous And Arterial Thrombosois In Pregnancy and puerperium.Neurological India:22:131

[8]. KingAB.Neurological conditions occurring as complication of pregnancy. Arch NeuralPsychiatry; 63:471

[9]. Prakashc, SinghS.CVT in puerperium.JAPI;8:363

[10]. SingB,PadmavathiS,PathakSN.Encephalopathyin pregnancy-a study of 36 cases.Neurol India1961;9:1

[11]. KrayenbuhlH.cerebral venous and sinus thrombosis.ClinNeurosurg 1967;14:1-24

[12]. JollySS,RaviB,SinghN.CVT in puerperium.JAPI1971;19:447 